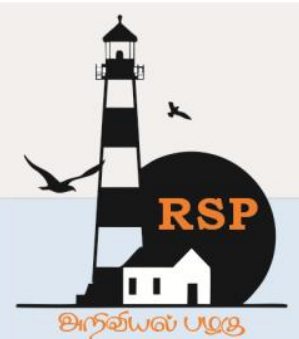

INTERNATIONAL RESEARCH JOURNAL ON ADVANCED SCIENCE HUB

\title{
Stress Intensity factors of a semi-elliptical crack in a Compressor blade of dovetail joint through numerical approach
}

Dr.Chandramouli Harige Ramaswamy ${ }^{1}$, Dr. Chakradhar Goud ${ }^{2}$

${ }^{1}$ Department of Mechanical Engineering, Shri JJT University, Jhunjhunu, Rajasthan, India.

${ }^{2}$ Professor \& Principal, Springfields Engineering College, Hyderabad, Telangana. India.

harigechandramouli@gmail.com ${ }^{1}$,cgsakki@gmail.com ${ }^{2}$

\begin{abstract}
Induced fatigue has led to a large number of deviations of service failures in a wide range of applications, where the deterioration of properties occurs due to the slipping between surfaces at a small displacement. In this article, a numerical simulation of a semi-elliptical crack in the dovetail joint of the compressor was performed to understand the destruction of the blade due to the stress contour in the contact area. A three-dimensional crack is modelled using the ANSYS Finite Element Analysis program, and the blade profile is modelled by CATIA V5R20 software. The study considers a model of an open semi-elliptical crack, and the parameters of the movement of the crack tip are estimated using three-dimensional singular elements of the crack tip in ANSYS. Initially, a three-dimensional finite element model of a compressor blade with a dovetail contact area was simulated in CATIA V5 and analysed in the ANSYS working environment to determine the maximum principal stresses in the crack-free region. After the crack front has been precisely identified and confirmed, fracture modelling is used to determine the impact of crack size with one side, as well as rotation speed, on fracture parameters (stress intensity factors).
\end{abstract}

Keywords: Dovetail joint, Elliptical crack, Stress Intensity factor, Finite element analysis

\section{Introduction:}

The root of the dovetail of the compressor blades is the case when the induced fatigue failure is a fairly common occurrence. Under the influence of centrifugal loading and vibration, fatigue occurs between the root of the blade, the disk and the stresses in the vicinity of the contact, which must be accurately estimated to predict the fatigue life. Stress assessment is a big problem for designers because of the complex geometry, load, and nonlinear behaviour.[1-4].Although considerable attention has been given in recent years to the analysis of failures of turbine blades with cracks, and also due to advances in 3D fracture analysis, efforts have been made to characterize the propagation of cracks in crack blades. In this study, the stress intensity factor is analysed based on the location where the theory of maximum principal stress is considered, and semi-elliptical cracks are modelled using the finite element method (FEM).[5-8]. P. Papanikos et al. conducted a three-dimensional nonlinear analysis by the finite element method of this area of the dovetail in the disk assemblies of the aircraft engine compressor using contact elements. Lucian witek (conducted a failure analysis of an aircraft engine turbine disk. Nonlinear finite element analysis was used to determine the stress state of the disk / blade assembly under various rotation conditions.[8-10]. 


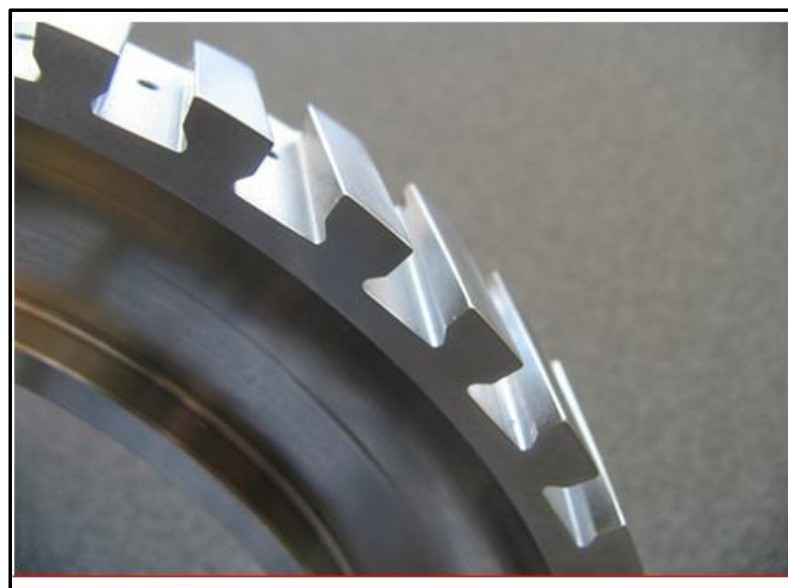

(a)

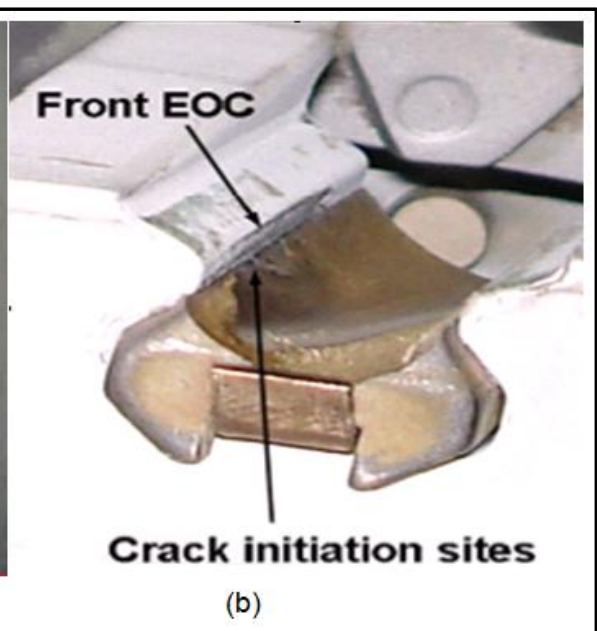

(b)

\section{Description of the problem}

Fig.1. Crack initiation sites on the compressor blade

In this study, a semi-elliptical crack is modelled based on maximum principal stress and Von mises stresses region on the compressor blade profile without crack. Then maximum Stress intensity factors for semi-elliptical crack at various contours are calculated for different crack lengths and rotation of the disc. Titanium alloy blade material is considered for analysis which is a good corrosion resistant, high chemical stability and excellent mechanical properties.

\section{Numerical simulation of compressor blade in}

\section{dovetail joint}

\subsection{Geometric modelling}

The geometric modelling was carried out using CATIA-V5. In view of disc symmetry only one sector of the blade was modelled for analysis as shown in the Fig. 2 and Fig.3. The element is defined by 10 nodes having three degrees of freedom at each node: translations in the nodal $\mathrm{x}$, $\mathrm{y}$, and $\mathrm{z}$ directions. For modelling semi elliptical crack, major radius of $0.05 \mathrm{~mm}$ and minor radius of $0.04 \mathrm{~mm}$ are considered.

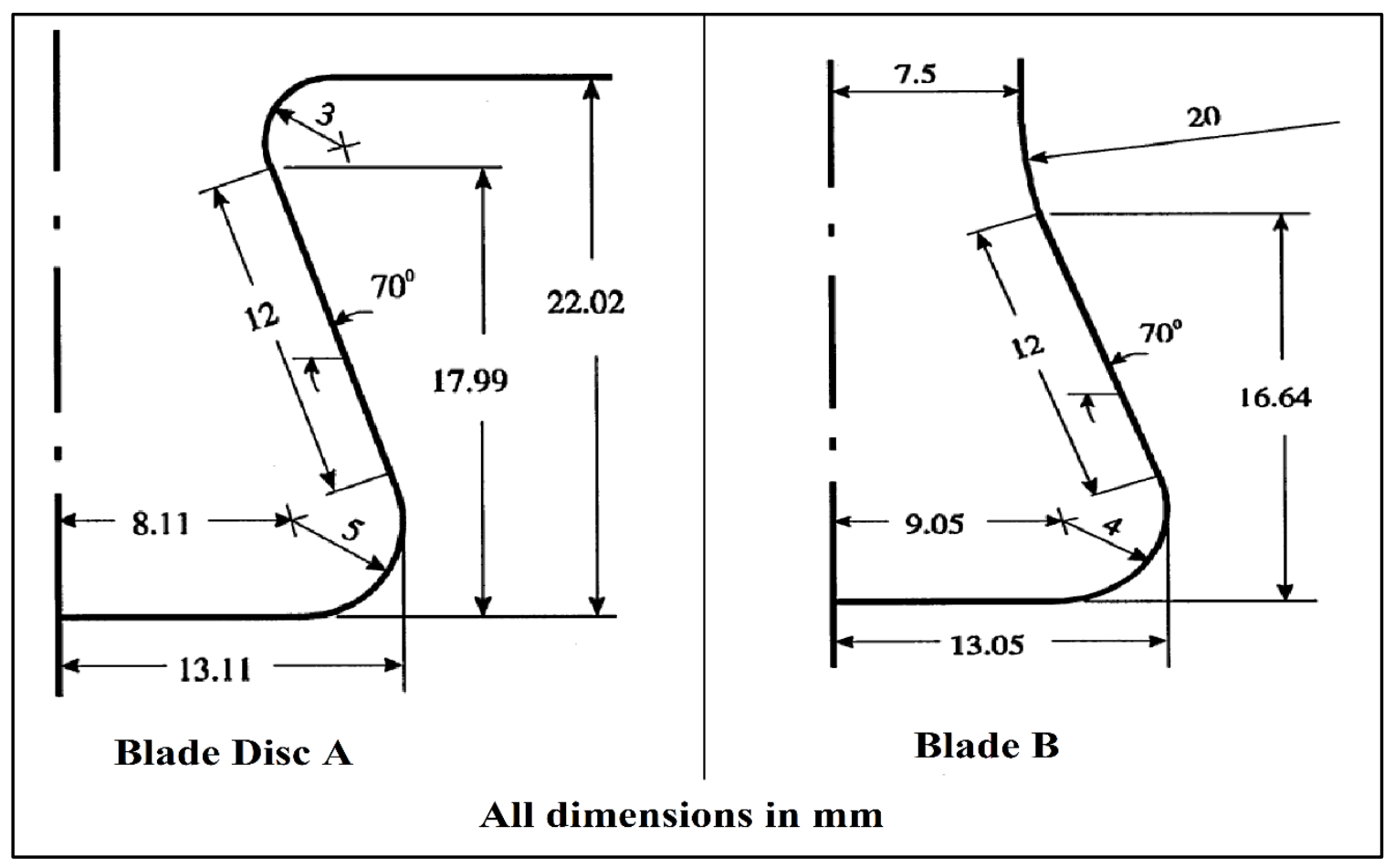

Fig.2.Geometric details of a compressor Blade dovetail joint 


\subsection{Finite Element modelling}

ANSYS software was used for the Discritization of the 3D model. Using SOLID187 components, the blade and disc were discretized. SOLID187 is a 10-node tetrahedral 3D structural element used to

\subsection{Contact properties}

To perform non-linear analysis, a symmetric contact was established from a solid to a solid contact of the blade and disk. CONTA174 and TARGET170 were used to represent the contact and target surfaces, respectively. The surface of the blade was modelled as a contact, and the surface of the disk was modelled as a target surface, as shown in Fig. 4. CONTA174 is used for three-dimensional structural and pair contact analysis. This element can be located on the surfaces of three-dimensional solid or shell elements that do not have any middle lateral nodes.

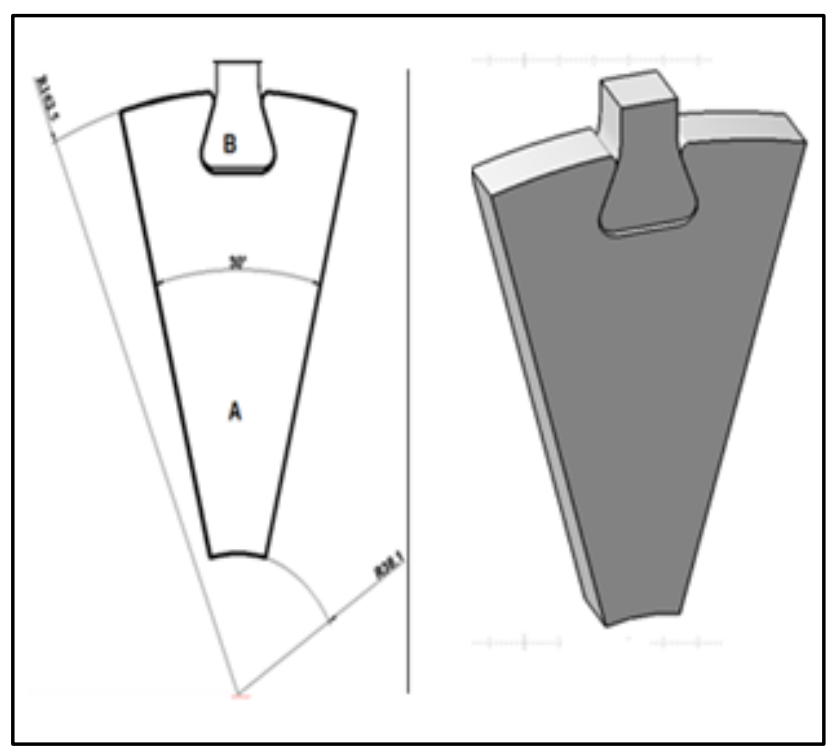

Fig. 3. Geometric details of a compressor blade dovetail joint 3D modeling

\subsection{Loads and Boundary Conditions}

To run the analysis only centrifugal load was considered. The boundary constraints are one of the most important factors in Finite element analysis. The boundary condition for compressor blade with dovetail joint is as shown in the Fig.6. The rotor and blade have cyclic symmetry about the axis hence only one sector of the model is considered for analysis. The bottom portion of the disc was constrained radially and the side faces of the disc were constrained using symmetry boundary conditions. model solid structures in three dimensions. Figure 4 depicts the finite element modelling of a compressor blade dovetail joint assembly and a semi-elliptical crack.

When the surface of an element penetrates one of the target elements of a segment on a given target surface, contact occurs. TARGET170 is a threedimensional target surface for linked contact elements that is used to describe various threedimensional target surfaces. The target surface is discretised by a set of elements of the target segment and connected to the corresponding contact surface through a common real constant set. In Fig.5, it's shown that there are total 113377 nodes, number of contact elements are 12113, number of solid elements are 66942 and number of total elements are 79056.

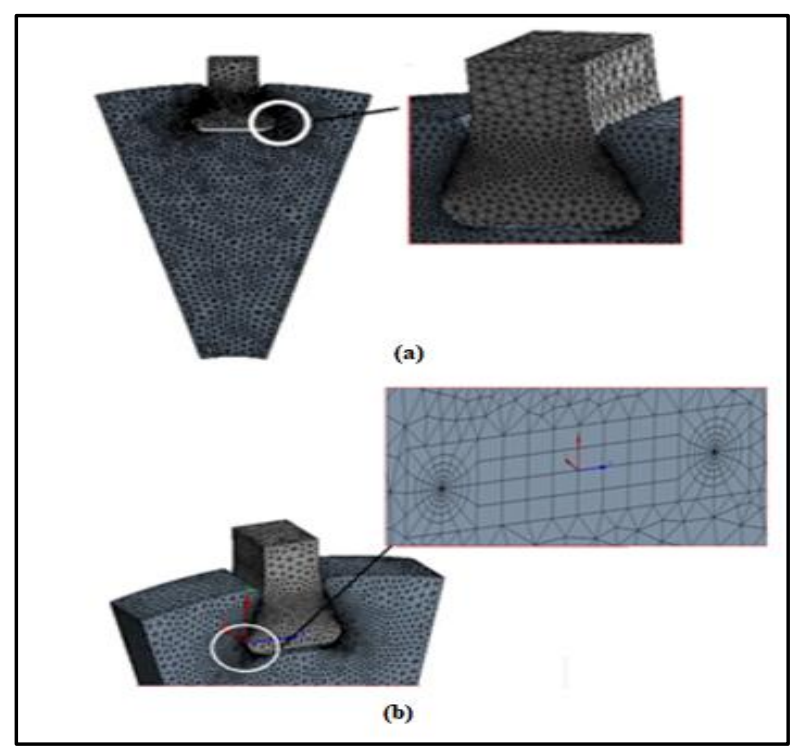

Fig.4. Finite element model (a) compressor dovetail joint (b) Semi-elliptical crack

\section{Results and discussions}

In this section, the results obtained from carrying out non-linear static analysis on 3-Dimensional dovetail joint of compressor are presented.

\subsection{Analysis of Compressor Blade dovetail joint without Crack}

In Fig.7. and Fig.8.show both the von-mises stress and the maximum principal stress at the contact region, which gives the identification of crack location at the contact region of the dovetail joint. 
www.rspsciencehub.com

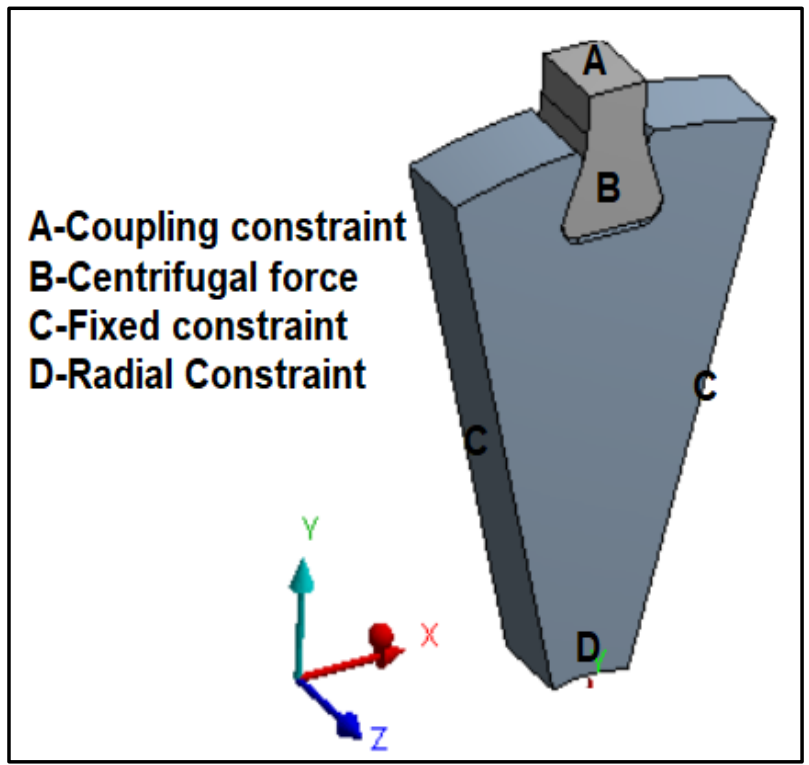

Fig.5. Modelling ofDovetail jointContact assembly

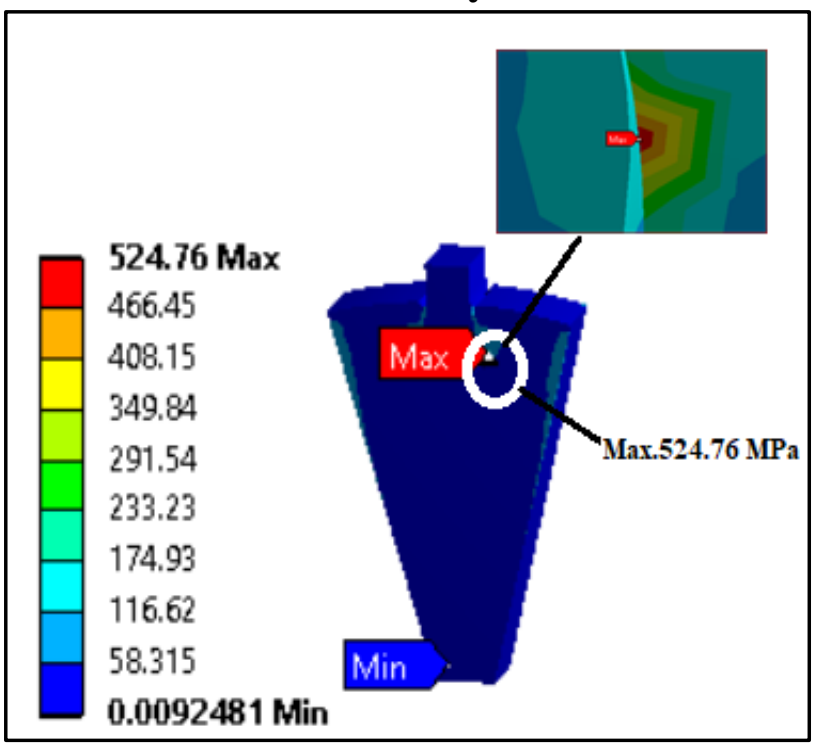

Fig.7. Maximum Von-mises stressat the contact region without crack

4.2 Analysis of Compressor Blade dovetail joint with Crack

Based on the results of the 3D analysis, high stressed location near the blade and disc contact region which is the probable location of initiation of crack was identified. In order to predict the stress Intensity factor, a semi-elliptical crack was introduced in the region where maximum vonmises stress and maximum principal stress occurs, which is shown in the fig.9 and Fig.10. The KCALC command used in plain strain option to calculate the maximum stress Intensity factor (K1) at the crack region, which is predominant, is shown in the Fig.11. From Fig.9, it's observed that von-mises stress is very high at the crack tip (823
Volume 03 Issue 04 April 2021

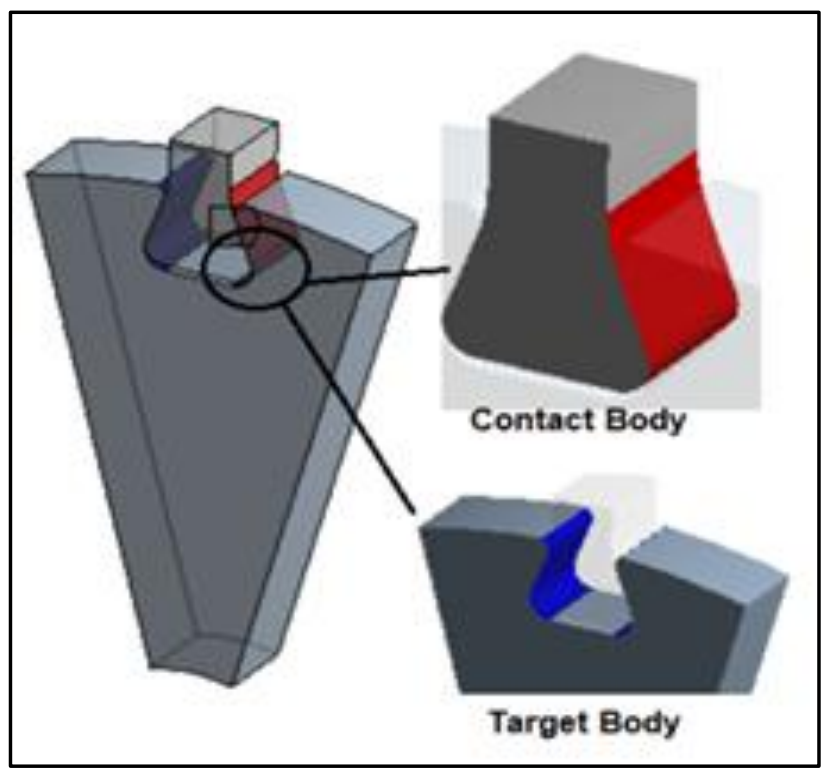

Fig. 6. Load and boundary Conditions of dovetail assembly

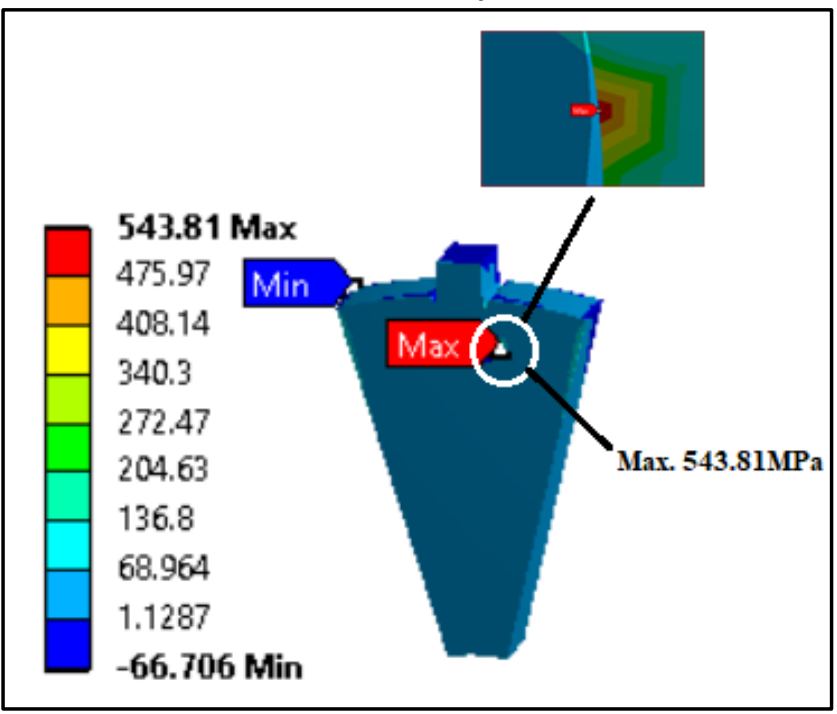

Fig.8. Maximum Principal Stress at the contact region without crack

MPa) because of singular elements and concentrated plastic zone at the crack tip. Maximum principal stress of $1339 \mathrm{MPa}$ is observed from the fig.10, which is higher than the ultimate stress value due to the crack growth rate. Fig. 11represents stress Intensity factors K1 for mode I.

\subsection{Evaluation of stress Intensity factor (SIF) for different modes in compressor disc.}

The results obtained from carrying out non-linear static analysis on 3D dovetail joint of compressor are presented here. From Fig.12, the results obtained are presented in terms of modes of I, II and III stress intensity factors (K1,K2 and K3) variations over various crack depth for semi- 
www.rspsciencehub.com

elliptical for a compressor speed of 8000 r.p.m ( $\omega$ $=837.75 \mathrm{rad} / \mathrm{s}$ ) for six contours. In Fig. 12. the values of stress Intensity factors for $\mathrm{K} 1, \mathrm{~K} 2$ and

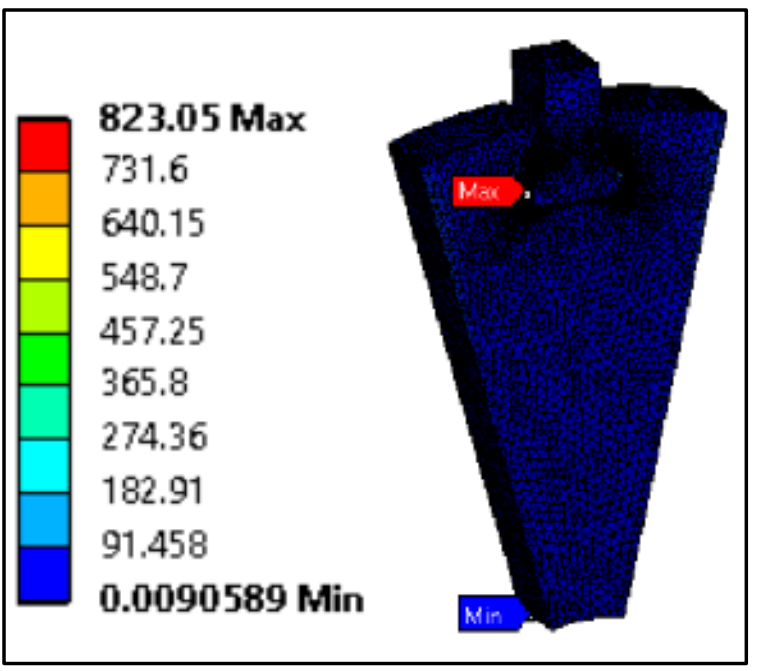

Fig.9 Maximum Von-mises stress at the crack region

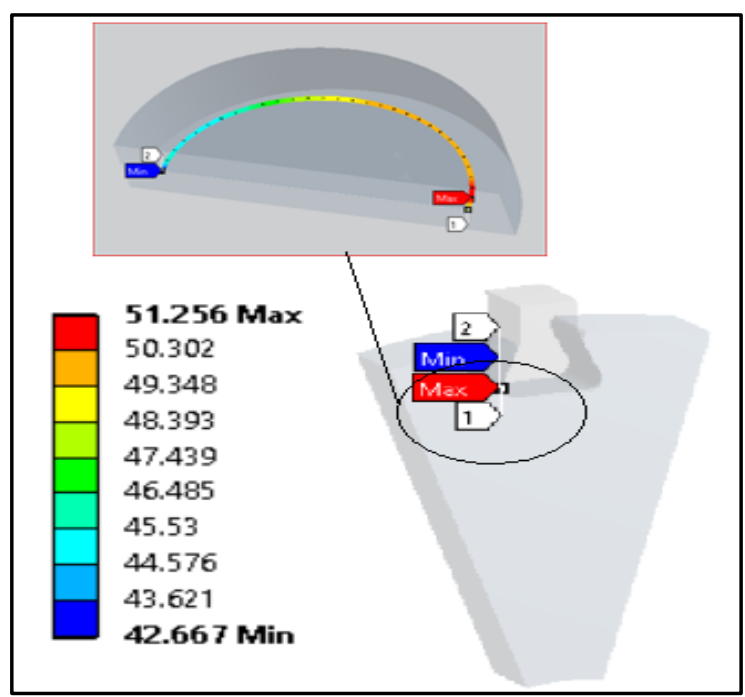

Fig.11 Maximum stress Intensity factor(K1) at

\section{Conclusions} the crack tip region

An effort has been made in the present study to understand the analysis of semi-elliptical crack in a compressor blade dovetail joint assembly through numerical simulation. Three-dimensional nonlinear finite element of a compressor blade dovetail joint assembly is carried out to evaluate the maximum stress in the contact region. The semi-elliptical crack analysis was carried out to find out the maximum stress Intensity factors for three fracture modes of different crack lengths. The SIF increases as the length of the crack depth increases. The Stress Intensity factor for a given
Volume 03 Issue 04 April 2021

$\mathrm{K} 3$ are plotted for fracture mode 1 , mode 2 and mode 3 respectively.

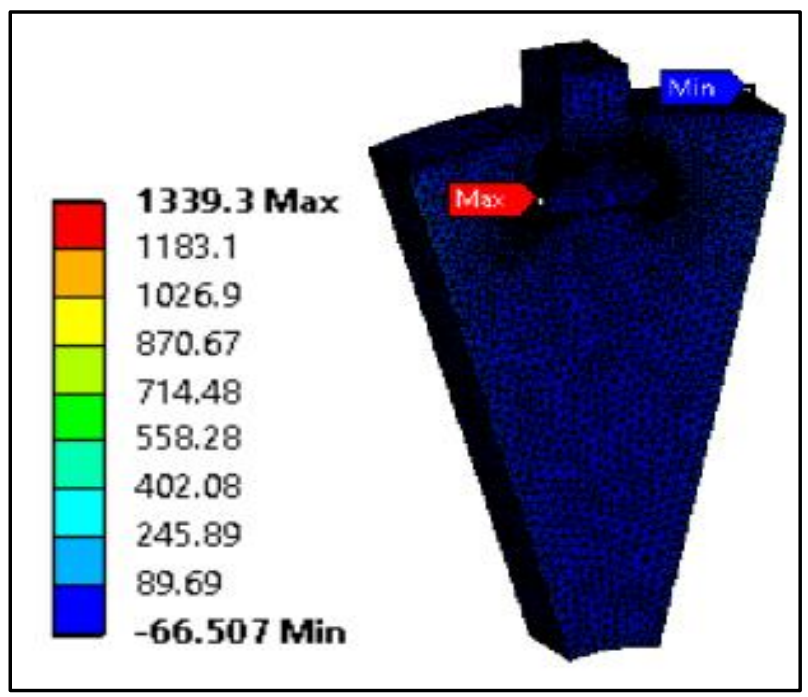

Fig.10 Maximum Principal Stress at the crack region

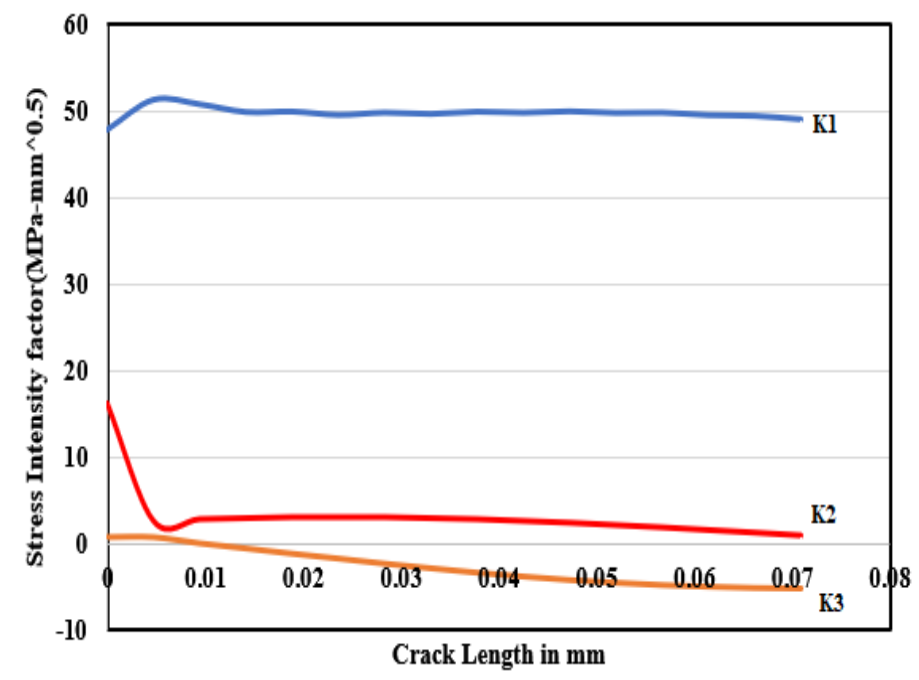

Fig.12 Values of K1, K2 and K3 for six contours

compressor speed is evaluated through the induced semi-elliptical crack is to predict the crack growth

\section{References}

Journals

[1].V. A. Vainshtok and I.V. Varfolomeyev, Stress intensity factor equations for part-elliptical cracks and their verification, Engineering Fracture Mechanics Vol. 34, No. I, pp. 125136, 1989.

[2]. A. Kermanpur a, H. Sepehri Amin, S. ZiaeiRad, N. Nourbakhshnia, M. Mosaddeghfar, "Failure analysis of Ti6Al4V gas turbine 
compressor blades", Engineering Failure Analysis, V 15, 2008, pp 1052-1064.

[3].P. Papanikos, S.A.Meguid and Z.Stjepanovic, "Three Dimensional Non-linear Finite Element Analysis of Dovetail joints in Aero-Engine disc", Finite Elements in analysis and Design, V29, 1998, pp 173-186.H.S.

[4].Hedia and S.M. Aldousari, "ThreeDimensional Finite Element Model for Evaluation the Stress Intensity Factors for Different Fracture Modes of Homogeneous Bimaterial", King Abdulaziz University, Jeddah, Saudi Arabia.

[5].LucjanWitek, "Failure Analysis of Turbine discs of an aero-engine" Engineering Failure analysis.V 13,2006,pp9-17.

[6]. R. Rajashekaran and D Nowell, "Fretting fatigue in Dovetail Blade Roots: Experiment and analysis", Tribology International, V 39, 2006, pp 1277-1285.Lucjan witek, "Failure analysis of Turbine discs of an Aero-engine", Engineering failure analysis V 13,2006,pp9-17.

[7].M.M.I. Hammouda, R.A. Pasha and A.S. Fayed, "Modelling of Cracking Sites/Development in Axial Dovetail Joints of Aero-Engine Compressor Discs", International Journal of Fatigue, V 29, 2007, pp 30-48.

\section{Book}

[8].Anderson, T.L., "Fracture Mechanics: Fundamentals and Applications," 3rd Edition

[9].David Broek, 'Elementary Engineering Fracture Mechanics', 1984, table 3.1, Pg 76.

[10]. Release 19.1 Documentation for ANSYS. 